\title{
Octadecadienoic Acid n-6 trans
}

National Cancer Institute

\section{Source}

National Cancer Institute. Octadecadienoic Acid n-6 trans. NCI Thesaurus. Code C68401.

A polyunsaturated long-chain fatty acid with an 18-carbon backbone and double bonds originating from the 6th position and the 8th, 9th, 10th, 11th, 12th or 13th positions from the methyl end. The bond geometry of the molecule induces a kink in the main axis of the aliphatic chain at the $n-6$ position. There are at least 8 different isomers that can be called by this name. 